\title{
Fibroadenomatoid mastopathy-like lesions arising in anogenital mammary-like glands in a 35-year-old man
}

\author{
Sergio Fernandez-Aguilar • Angel Vazquez-Martín
}

Accepted: 2 April 2009/Published online: 24 April 2009

(C) Springer-Verlag 2009

\section{Dear Editor,}

Anogenital mammary-like glands were first described by Hartung in 1872 as "ectopic breast tissue." Over a century later, van der Putte defined these structures as a new variant of cutaneous glands that occurs in the anogenital region, most highly concentrated in the interlabial sulcus of the vulva, which explain a series of disorders of the anogenital region in women. These lesions show a striking resemblance to those of an eutopic breast tissue and include fibroadenoma, phyllodes tumor, and adenosis tumor. We report a lesion that occurred in the anogenital area of a man and apparently arose in anogenital mammary-like glands.

A 35-year-old man presented with pain in the anogenital area. Past medical history was unremarkable, and he was in good physical condition. In particular, there was no history of prior malignancy or breast disease. Clinical examination showed a perianal lesion macroscopically consistent with fistula, which was afterward surgically removed. Macroscopic examination revealed a partially ulcerated cutaneous specimen with a $1-\mathrm{cm}$ long fistula. No other gross lesion was seen. Tissue was formalin-fixed and paraffinembedded. Microscopic examination showed a focally ulcerated epidermis with an underlying inflammatory fistula. Adjacent to the fistula, individual lobules and groups of lobules with the appearance of miniature fibroadenomas were noted. The epithelial component revealed mainly flat to cuboidal, non-atypical cells with clear cytoplasm lined by spindle myoepithelial cells. Some apocrine, sweat duct-like structures were present within the lobules. The intralobular stroma showed a periductal, concentric distribution and fibromyxoid, amphophilic appearance. Immunostaining using the avidin-biotin peroxidase complex $(\mathrm{ABC})$ was performed using antibodies against the following antigens: estrogen receptor, progesterone receptor, and androgen receptor. Appropriate positive and negative controls were obtained for each antibody. Fibroadenomatoid structures showed focal positivity for androgen receptor and were negative for both estrogen and progesterone receptors.

Fibroadenomatoid mastopathy originating from anogenital mammary-like glands is rare. To date, cases reported in the literature mostly concern vulval lesions documented in women. To the best of our knowledge, we describe the first case of fibroadenomatoid mastopathy of anogenital mammary-like glands in a male patient. This case illustrates that it is important to have in mind the presence of anogenital mammary-like glands because they may give rise to a variety of benign and malignant lesions. We also believe that these lesions occurring in men, when benign, could be underreported in the literature. 